\section{Identifying patients at risk from nonalcoholic fatty liver-related hepatocellular carcinomas}

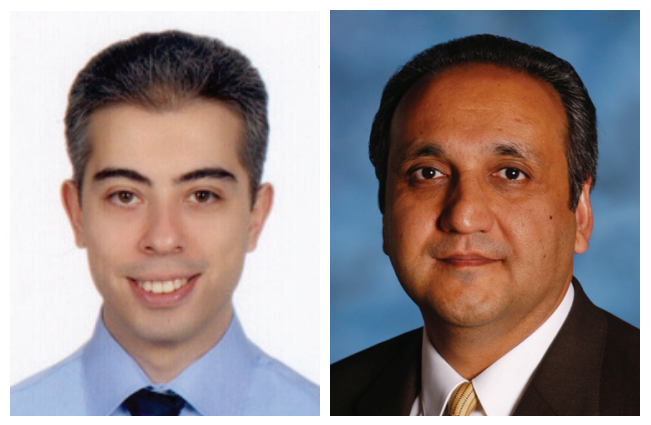

\author{
“Nonalcoholic fatty liver \\ disease is the most prevalent \\ form of chronic liver disease \\ and is increasingly recognized \\ as the hepatic manifestation of \\ metabolic syndrome."
}

Mehmet Sayiner ${ }^{1} \&$ Zobair M Younossi ${ }^{*}, 1,2$

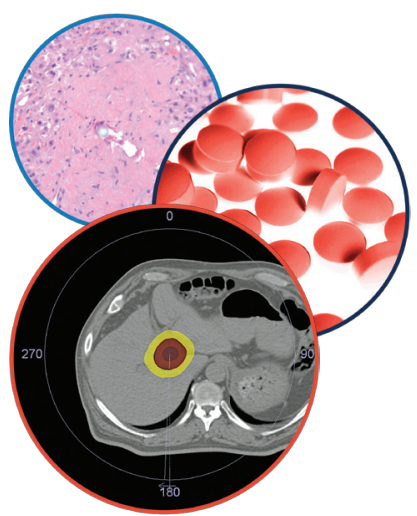

Hepatic Oncology

First draft submitted: 5 February 2016; Accepted for publication: 8 February 2016; Published online: 23 March 2016

Nonalcoholic fatty liver disease (NAFLD) is the most prevalent form of chronic liver disease and is increasingly recognized as the hepatic manifestation of metabolic syndrome [1,2]. NAFLD is a spectrum of clinical and pathologic entities, which includes patients with hepatic steatosis in the absence of alcohol or other secondary causes of fatty liver. Histologically, NAFLD covers a broad range from simple steatosis to nonalcoholic steatohepatitis (NASH), in which hepatocyte damage accompanies hepatic steatosis. There is compelling evidence that NASH is the subtype of NAFLD that increases the risk of cirrhosis and its complications [1-3]. Over the past decade, hepatocellular carcinoma has become recognized as an important complication of NAFLD-related cirrhosis.

The incidence of hepatocellular carcinoma (HCC), which represents the $80-90 \%$ of all primary liver cancers, has been increasing in the last decades. In
USA, the incidence of HCC increased from 1.5 to 4.9 per 100,000 individuals in the last three decades [4]. Major risk factors for HCC include viral hepatitis, alcoholic liver disease, NAFLD and other types of chronic liver disease. Additionally, male gender, older age, smoking, obesity, Type 2 diabetes and metabolic syndrome have been associated with increased risk for HCC [5]. Although viral hepatitis is the most common cause of HCC globally, recent data suggest that NAFLD may be becoming another important cause of HCC, especially in the western world, where the prevalence of obesity-related NAFLD has been increasing [6].

The majority of HCC cases occur in the seedbed of cirrhosis (nearly 70-85\%) and a third of cirrhotic patients develop HCC during their lifetime. In a recent study, the annual cumulative incidence of HCC was $2.6 \%$ among NAFLD-associated cirrhosis, whereas it was $4 \%$ in patients with

\section{KEYWORDS}

- hepatocellular carcinoma - NAFLD

- NASH • risk factors • surveillance

“...in addition to cirrhosis, metabolic conditions may be playing an important role in promoting hepatocellular carcinoma.”

'Betty \& Guy Beatty Center for Integrated Research, Inova Health System, Falls Church, VA, USA

${ }^{2}$ Center For Liver Disease, Department of Medicine, Inova Fairfax Hospital, Falls Church, VA, USA

*Author for correspondence: Tel.. +1 703776 2540; Fax: +1 703776 4386; zobair.younossi@inova.org

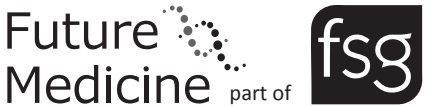


“...because of the increased prevalence of obesity and metabolic syndrome, nonalcoholic fatty liver disease has become an important cause of chronic liver disease and its complications including hepatocellular carcinoma.”
HCV-associated cirrhosis [4]. It has been recently recognized that $\mathrm{HCC}$ can occur in patients with NAFLD without cirrhosis [7]. In fact, in a recent study of patients with HCC, diagnosis of NAFLD was independently associated with presence of HCC in the noncirrhotic livers [8]. Interestingly, in a study by Piscaglia et al. cirrhosis was present in only $50 \%$ of NAFLD-HCC patients and nearly all HCV-HCC patients [9]. Furthermore, in another study from Veterans Administration (VA) $13 \%$ of patients with HCC did not have cirrhosis, and the relative risk of having HCC in the absence of cirrhosis was 5.4-times for NAFLD and five-times for those with metabolic syndrome [10].

These data suggest that in addition to cirrhosis, metabolic conditions may be playing an important role in promoting HCC. In fact, accumulating data indicate that among patients with NAFLD, the risk of HCC is higher in overweight and obese patients [11-14]. In a metaanalysis by Larsson et al., the risk of liver cancer was 17 and $89 \%$ higher in the overweight and obese patients, respectively, compared with individuals with normal weight [15]. In another recent systematic review of ten cohort studies, the association between obesity and HCC risk was confirmed with the relative risk of HCC ranging from 1.4 to 4.1 [11].

In addition to obesity, Type 2 diabetes has also been independently associated with a 2.3-fold increase in the risk of HCC $[12,14]$. Additionally, this risk seems to be two- to fourtimes higher among men with diabetes [5]. Based on the overwhelming data that diabetes is also an independent risk factor for both NAFLD and HCC, effective treatment of diabetes and insulin resistance may have an impact on the future incidence of HCC. Nevertheless, future studies are needed to provide evidence supporting this treatment strategy and its impact on the prevention of HCC.

Finally, hepatic iron overload has been postulated to play a role in the pathogenesis of HCC. Previous studies revealed that among NAFLD patients, liver iron depots were larger and more frequent in those who developed HCC as compared with those who did not [16]. On the other hand, other studies have contradictory evidence regarding the role of iron in $\mathrm{HCC}[14,16]$. At the moment, the role of iron in the pathogenesis of HCC remains controversial.

In addition to the environmental, viral and host factors associated with HCC, there are recent data on the genetic susceptibility to HCC. In this context, a single nucleotide polymorphism in adiponutrin gene (PNPLA3), I148M, was not only associated with NAFLD but also linked to liver fibrosis and the development of HCC [17]. Future validation of these genomics tests will be important as a tool of personalized medicine to identify patients at higher risk for HCC.

In addition to identifying risks associated with HCC, it is also important to summarize the current recommendations regarding screening for HCC. In 2016, most guidelines suggest that screening for HCC is effective and cost effective $[18,19]$. In this context, cirrhosis remains the primary indication for HCC surveillance [19]. The recommended surveillance is based on ultrasound examination with a screening interval of 6 months. Nevertheless, ultrasound may fail to detect small tumors in patients with NAFLD due to central obesity and increased hepatic fat. Therefore, in individuals at risk for HCC with suboptimal ultrasound testing, dynamic imaging with magnetic resonance should be considered. Although widely used in clinical practice, $\alpha$-fetoprotein lacks adequate sensitivity and specificity for effective surveillance for HCC.

Although all cirrhotics must be screened for HCC, patients with $\mathrm{HBV}$ who are not cirrhotic are also at increased risk for HCC. Furthermore, the increased risk for HCC in noncirrhotics with NAFLD is intriguing. On the other hand, it is likely that the number of patients with noncirrhotic NAFLD who develop HCC is small. Nevertheless, given the epidemiologic burden of NAFLD, the number of patients with noncirrhotic NAFLD who could present with HCC could become larger. Despite these intriguing data, in 2016, there are no formal recommendations about screening these patients for HCC.

In conclusion, because of the increased prevalence of obesity and metabolic syndrome, NAFLD has become an important cause of chronic liver disease and its complications including HCC. To identify and follow patients who are at risk of NAFLD-related HCC, metabolic conditions like obesity, Type 2 diabetes and insulin resistance should be comprehensively assessed as well as the family history. Patients with NASH should be followed-up carefully for development and progression to cirrhosis. With the access to highly effective treatments for viral hepatitis, its role in HCC development is expected to diminish over the coming decades, causing a global shift 
in the epidemiology of HCC and raising the importance of metabolic risk factors for NAFLD in the global burden of HCC.

Financial \& competing interests

disclosure

The authors have no relevant affiliations or financial involvement with any organization or entity with a

\section{References}

1 Golabi P, Sayiner M, Fazel Y et al. Current complications and challenges in nonalcoholic steatohepatitis screening and diagnosis. Expert Rev. Gastroenterol. Hepatol. 10, 63-71 (2015).

2 Younossi ZM, Koenig AB, Abdelatif D et al. Global epidemiology of non-alcoholic fatty liver disease-meta-analytic assessment of prevalence, incidence and outcomes. Hepatology doi:10.1002/hep.28431 (2015) (Epub ahead of print).

3 Mishra A, Younossi ZM. Epidemiology and natural history of non-alcoholic fatty liver disease. J. Clin. Exp. Hepatol. 2, 135-144 (2012).

4 Baffy G, Brunt EM, Caldwell SH. Hepatocellular carcinoma in non-alcoholic fatty liver disease: an emerging menace. J. Hepatol. 56, 1384-1391 (2012).

5 Dongiovanni P, Romeo S, Valenti L. Hepatocellular carcinoma in nonalcoholic fatty liver: role of environmental and genetic factors. World J. Gastroenterol. 20, 12945-12955 (2014)

6 Younossi ZM, Otgonsuren M, Henry L et al. Association of Nonalcoholic Fatty Liver Disease (NAFLD) with hepatocellular carcinoma (HCC) in the United States from 2004 to 2009. Hepatology 62, 1723-1730 (2015). financial interest in or financial conflict with the subject matter or materials discussed in the manuscript. This includes employment, consultancies, honoraria, stock ownership or options, expert testimony, grants or patents received or pending, or royalties.

No writing assistance was utilized in the production of this manuscript.

7 Ertle J, Dechene A, Sowa JP et al. Nonalcoholic fatty liver disease progresses to hepatocellular carcinoma in the absence of apparent cirrhosis. Int. J. Cancer 128, 2436-2443 (2011).

8 van Meer S, van Erpecum KJ, Sprengers D et al. Hepatocellular carcinoma in cirrhotic versus noncirrhotic livers: results from a large cohort in The Netherlands. Eur. J. Gastroenterol. Hepatol. 28, 352-359 (2016).

9 Piscaglia F, Svegliati-Baroni G, Barchetti A et al. Clinical patterns of hepatocellular carcinoma (HCC) in non alcoholic fatty liver disease (NAFLD): a multicenter prospective study. Hepatology doi:10.1002/hep.28368 (2015) (Epub ahead of print).

10 Mittal S, Sada YH, El-Serag HB et al. Temporal trends of nonalcoholic fatty liver disease-related hepatocellular carcinoma in the veteran affairs population. Clin. Gastroenterol. Hepatol. 13(3), 594-601.e1 (2015).

11 Saunders D, Seidel D, Allison M et al. Systematic review: the association between obesity and hepatocellular carcinoma - epidemiological evidence. Aliment. Pharmacol. Ther. 31, 1051-1063 (2010).

12 Baffy G. Editorial: hepatocellular carcinoma in Type 2 diabetes: more than meets the eye. Am. J. Gastroenterol. 107, 53-55 (2012).
13 Vongsuvanh R, van der Poorten D, George J. Non-alcoholic fatty liver disease-related hepatocellular carcinoma: a sleeping tiger in the Asia Pacific. Hepatol. Int. 7(Suppl. 2), 823-832 (2013).

14 Pocha C, Kolly P, Dufour JF. Nonalcoholic fatty liver disease-related hepatocellular carcinoma: a problem of growing magnitude. Semin. Liver Dis. 35, 304-317 (2015).

15 Larsson SC, Wolk A. Overweight, obesity and risk of liver cancer: a meta-analysis of cohort studies. Br. J. Cancer 97, 1005-1008 (2007).

16 Sorrentino P, D’Angelo S, Ferbo U et al. Liver iron excess in patients with hepatocellular carcinoma developed on non-alcoholic steato-hepatitis. J. Hepatol. 50, 351-357 (2009).

17 Liu YL, Patman GL, Leathart JB et al. Carriage of the PNPLA3 rs738409 C > G polymorphism confers an increased risk of non-alcoholic fatty liver disease associated hepatocellular carcinoma. J. Hepatol. 61, 75-81 (2014).

18 EASL. EASL clinical practice guidelines. www.easli.eu

19 Bruix J, Sherman M. Management of hepatocellular carcinoma: an update. Hepatology 53, 1020-1022 (2011). 